defined as "positive" since the uptake was higher than liver, and twelve/thirty $(52.2 \%)$ were defined as "negative" since the uptake was lower than liver, regardless of SUVs and clinical manifestations. A semi-quantitative analysis assessed whether the values of the SUVmax BM/liver were higher than the cut-off of 2.09 in "positive" PET/MR and lower in the "negative" ones and if the clinical manifestations were present or absent in agreement with the evaluation of SUVs for each patient. BM was found to be active (SUVmax ratio > of 2.09) in 7 out of 11 patients when the PET/MR was defined "positive", while only in 1 case out of 12 BM SUVmax was $>2.09$ when the exam was "negative." Clinical manifestations were present in 10 out of 11 AOSD with a "positive" scan and in 7 out of 11 with both a "positive" scan and a SUV max BM/liver >2.09. Clinical manifestations were present in 1 out of 12 patients with a "negative" scan, while in 10 out of 12 cases with both a negative scan and a SUV max BM/liver <2.09 were absent. Six patients repeated PET/MR during follow-up. The values of the SUVmax BM/ liver significantly decreased after anti IL-1 $\beta$ treatment with anakinra. In two cases in which anakinra was deferred, the BM SUVmax values exceeded the cut-off of 2.09 despite the patients did not complain any symptom or inflammation markers increase.

Conclusion: ${ }^{18} \mathrm{~F}$ FGD-PET/MR could be able to evaluate the disease activity in AOSD when clinical manifestations and serum markers are not sufficient to establish it. The uptake on BM seems quite sensitive in pointing out the disease severity and in assessing the response to anti IL-1 $\beta$ therapy. ${ }^{18} \mathrm{~F} \mathrm{PET/MR}$ is an accurate and repeatable method, however further studies are required to validate its applicability in routinary clinical practice.

Disclosure of Interests: None declared

DOI: 10.1136/annrheumdis-2020-eular.5747

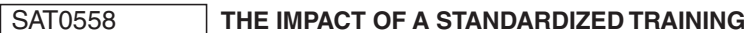 PROGRAM FOR IMPROVING THE RELIABILITY AND AGREEMENT - A STUDY OF VASCULAR ULTRASOUND FOR DIAGNOSING GIANT CELL ARTERITIS IN DENMARK}

S. Chrysidis $^{1,2,3}$, L. Terslev ${ }^{4}$, R. Christensen ${ }^{2,3}$, U. Fredberg ${ }^{2,5}$, K. Larsen ${ }^{1,2}$, T. Lorenzen ${ }^{5}$, U. Møller Døhn ${ }^{4}$, A. Diamandopoulos ${ }^{6} .{ }^{1}$ Hospital of Southwest Jutland, Esbjerg, Denmark; ${ }^{2}$ University, of Southern Denmark, Denmark; ${ }^{3}$ The Parker Institute, Copenhagen, Denmark; ${ }^{4}$ Rigshospitalet, Glostrup and COPECARE, Copenhagen, Denmark; ${ }^{5}$ Silkeborg Regional Hospital, Aarhus University, Denmark; ${ }^{6}$ Martina Hansen Hospital, Baerum, Norway

Background: Due to a high level of evidence of good test performance, accessibility, minimal invasiveness, low cost, and good overall performance, EULAR recommends ultrasound (US) of the temporal and axillary arteries as primary diagnostic imaging test in patients suspected of Giant Cell Arteritis (GCA) (1).

Despite the growing body of evidence supporting the utility of US in GCA, standardized training programs and their impact on reliability are lacking(1). In TABUL study (2), the only US study published to date using a standardised US training program, the interobserver agreement by 12 different sonographers was only moderate, illustrating the challenges presented in the education for US in GCA. Objectives: To evaluate the impact of a standardized training program including equipment adjustment on the agreement and reliability of US in the diagnosis of GCA for experienced musculoskeletal (MSK)ultrasonographers, without previous experience on vascular US.

Methods: Five rheumatologists with long-standing experience in MSK US were trained by a standardized training program including equipment adjustment (Box 1) prior to a prospective, non-interventional observational study in patients suspected having GCA.The rheumatologist performing the US subsequently evaluated the images blinded to the patients data. Thereafter the images were evaluated by a blinded external expert (i.e. considered the gold standard).

Results: In three Danish centers 112 patients were included, 59\% females, mean age 72.4 (SD) 7.9 years and median CRP 55 (IQR 21-100)mg/l. Median duration of prednisolone treatment prior to US examination was 0 (IQR 0 to 1) days. $92 \%$ of the patients reported a newly emerged localized headache.

The reliability between the performing ultrasonographer and the US expert for the overall GCA diagnosis, as for the diagnosis of cranial (c-GCA) and large-vessel GCA (Lv-GCA) was excellent. In addition, excellent reliability was also found for the US examination of all examined arteries (Table 1). According to the US expert, vasculitis changes were found in 66 patients with the pathological findings distributed as presented in table 1.
Tabel 1

(1)

\begin{tabular}{|c|c|c|c|c|}
\hline Variables & $\begin{array}{l}\text { Pathological } \\
\text { findings (\%) }\end{array}$ & $\begin{array}{l}\text { Interobserver } \\
\text { agreement (\%) }\end{array}$ & $\begin{array}{c}\text { Interobserver } \\
\text { Reliability } \\
\text { Kappa Coefficient }\end{array}$ & $\begin{array}{l}95 \% \text { Confidence } \\
\text { Limits }\end{array}$ \\
\hline US positive for GCA & $\begin{array}{c}59 \% \\
66 / 112\end{array}$ & $96 \%$ & 0.93 & $0.85-0.99$ \\
\hline US positive for CGCA & $\begin{array}{c}53 \% \\
59 / 112\end{array}$ & $95 \%$ & 0.89 & $0.81-0.98$ \\
\hline US positive for Lv-GCA & $\begin{array}{c}19 \% \\
21 / 112\end{array}$ & $96 \%$ & 0.89 & $0.78-0.995$ \\
\hline $\begin{array}{l}\text { Halo sign TA, all } \\
\text { segments }\end{array}$ & $\begin{array}{c}51 \% \\
57 / 112\end{array}$ & $96 \%$ & 0.91 & $0.83-0.99$ \\
\hline $\begin{array}{l}\text { Compression sign TA, } \\
\text { all segments }\end{array}$ & $\begin{array}{c}48 \% \\
51 / 107\end{array}$ & $94 \%$ & 0.89 & $0.80-0.98$ \\
\hline Halo sign FA & $\begin{array}{c}20 \% \\
23 / 112\end{array}$ & $96 \%$ & 0.87 & $0.75-0.98$ \\
\hline $\begin{array}{l}\text { Compression sign } \\
\text { FA }\end{array}$ & $\begin{array}{c}16 \% \\
17 / 107\end{array}$ & $96 \%$ & 0.86 & $0.73-0.99$ \\
\hline Halo sign AA & $\begin{array}{c}18 \% \\
20 / 112\end{array}$ & $97 \%$ & 0.91 & $0.81-1.00$ \\
\hline Halo sign $A C$ & $\begin{array}{l}4 \% \\
6 / 11\end{array}$ & $100 \%$ & 1.00 & $1.00-1.00$ \\
\hline
\end{tabular}

TA: Temporal; FA: Facial; AA: Axillary; AC: common Carotid, artery

Conclusion: Our training program resulted in excellent reliability of US findings in patients suspected of having GCA and for the final diagnosis. The training program could be used when implementing vascular US in clinical practice. Box 1

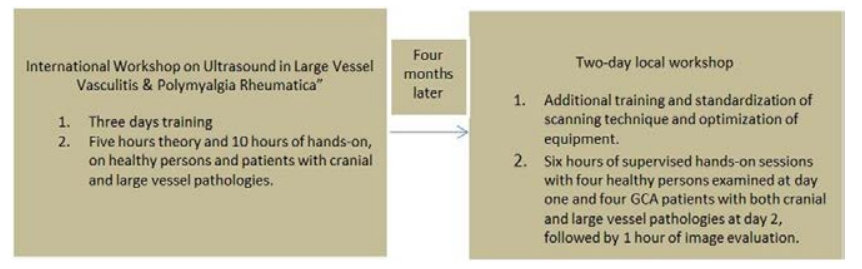

References:

[1] Dejaco $C$ et al. EULAR recommendations for the use of imaging in large vessel vasculitis in clinical practice. Ann Rheum Dis 2018;77:636

[2] Luqmani $\mathrm{R}$ et al. The Role of Ultrasound Compared to Biopsy of Temporal Arteries in the Diagnosis and Treatment of Giant Cell Arteritis: a diagnostic accuracy and cost-effectiveness study Health Technol Assess 2016;20:1_238

Disclosure of Interests: stavros chrysidis: None declared, Lene Terslev Speakers bureau: LT declares speakers fees from Roche, MSD, BMS, Pfizer, AbbVie Novartis, and Janssen., Robin Christensen: None declared, Ulrich Fredberg: None declared, Knud Larsen: None declared, Tove Lorenzen: None declared, Uffe Møller Døhn: None declared, Andreas Diamandopoulos: None declared DOI: 10.1136/annrheumdis-2020-eular.3274

\section{SAT0559 INCREASED FREQUENCY OF INTER- AND SUBMETATARSAL BURSITIS AND MORTON'S NEUROMA IN RHEUMATOID ARTHRITIS: RESULTS OF A LARGE CASE-CONTROLLED MRI STUDY OF FOREFEET IN PATIENTS WITH EARLY ARTHRITIS AND HEALTHY CONTROLS}

Y. Dakkak ${ }^{1}$, E. Niemantsverdriet ${ }^{1}$, A. Van der Helm - van Mil ${ }^{1,2}$, M. Reijnierse ${ }^{3}$.

${ }^{1}$ Leiden University Medical Center (LUMC), Rheumatology, Leiden,

Netherlands; ${ }^{2}$ Erasmus MC, Rheumatology, Rotterdam, Netherlands; ${ }^{3}$ Leiden

University Medical Center (LUMC), Radiology, Leiden, Netherlands

Background: The forefoot is a preferential location for tendon and joint inflammation in rheumatoid arthritis (RA). Some imaging studies suggested that intermetatarsal and submetatarsal pathology (such as bursitis and Morton's neuroma) are also involved in RA, but these studies were small and its association was not thoroughly explored.

Objectives: To determine whether intermetatarsal bursitis (IMB), Morton's neuroma $(\mathrm{MN})$ and submetatarsal bursitis (SMB) occur more often in early RA compared to patients with other early arthritides and healthy controls. Contrast-enhancement in the subcutis that has been described as diffuse submetatarsal alterations (DSMA) were also included.

Methods: In this cross-sectional cohort-study, consecutive patients with RA, other arthritides and healthy controls underwent MRI of unilateral forefoot. Two readers, a trained $\mathrm{PhD}$-student an experienced MSK-radiologist, scored IMB, MN, SMB and DSMA in consensus, and measured transverse and dorsoplantar 
diameters of IMB, MN and SMB. Logistic regression models determined their association with RA, and test characteristics for RA were calculated. Lesionsizes were plotted.

Results: 634 participants underwent MRI: 157 consecutive patients with RA

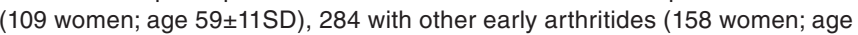
$56 \pm 17 S D$ ), and 193 healthy controls (136 women; age 50 $\pm 16 S D$ ). Univariably, $I M B, M N$ and SMB were more prevalent in RA (all $P<0.001$ ), DSMA was not $(P=0.16)$. Multivariably, $M B, S M B$ and $M N$ were all associated with $R A$ independent of each other $(P<0.016)$. IMB was most frequent (sensitivity 69\%), followed by SBM and MN (25\% and 19\%), specificity was high $(70 \%, 96 \%$, $94 \%$ respectively compared to other arthritides and $84 \%, 99 \%$ and $97 \%$ compared to healthy controls).

Although IMB, MN and SNB were more frequent in RA, the lesion-size was mainly similar in all groups. For $\mathrm{MN}$ a dorsoplantar diameter $>6 \mathrm{~mm}$ or transverse diameter $>5 \mathrm{~mm}$ was highly specific (specificity $100 \%$ compared to healthy controls), however it was infrequent (sensitivity $12 \%$ and $13 \%$, respectively). For IMB and SMB no cut-off size could be distinguished with high specificity.

Conclusion: Intermetatarsal bursitis, Morton's neuroma and submetatarsal bursitis are increased prevalent in early RA and could be considered as disease features.

Disclosure of Interests: None declared

DOI: 10.1136/annrheumdis-2020-eular.2107

\section{\begin{tabular}{l|l}
\hline SAT0560 & THE PROGNOSTIC VALUE OF ULTRASONOGRAPHIC
\end{tabular} FINDINGS IN INDIVIDUALS WITH ASYMPTOMATIC HYPERURICEMIA}

W. Li ${ }^{1}$, W. Fan ${ }^{1}$, J. Zhu ${ }^{1}$, Z. Chen ${ }^{1}$, F. Liu ${ }^{1} .{ }^{1}$ Peking University People's Hospital, Ultrasound, Beijing, China

Background: Chronic and steady asymptomatic hyperuricemia (AHU) can eventually lead to the deposition of monosodium urate crystals in joints and soft tissues. The rate of progression from AHU to clinically evident gout varies and mainly depends on serum uric acid levels. However, little is known about the prognostic value of ultrasonographic findings in individuals with AHU in detail. Objectives: To explore the prognostic value of ultrasonographic findings in individuals with asymptomatic hyperuricemia.

Methods: We analyzed the ultrasonographic findings (snowstorm sign, double-contour (DC) sign, tophi, bone erosion, and abnormal blood flow) of bilateral knees, ankles and the first metatarsal-phalangeal joints (1st MTP) of individuals with AHU at Peking University People's hospital between June 2014 and May 2016. All individuals were followed up for two years.

Results: Among 218 individuals with $\mathrm{AHU}$, the prevalence of snowstorm sign, DC sign, tophi, bone erosion and abnormal blood flow was $41 \%, 23 \%, 4 \%, 9 \%$ and $13 \%$, respectively. Gout attacked in 36 patients during 2-year follow-up with 4.5 years of HU duration. The first attack affected the 1st MTP in $60 \%$, the ankle in $31 \%$, and the knee in $11 \%$ of the patients with gout. Patients with gout attack has longer hyperuricemia duration compared with individuals with AHU without gout attack. DC sign, tophi, and bone erosion on ultrasound were more frequently presented in patients with gout attack compared with individuals with AHU without gout attack. However, the prevalence of snowstorm sign and and abnormal blood flow on ultrasound has no significant differences between patients with gout attack and individuals with $\mathrm{AHU}$ without gout attack.

Conclusion: Longer hyperuricemia duration, DC sign, tophi, and bone erosion on ultrasound in individuals with AHU could be associated with gout attack. References:

[1] Neogi T, Jansen TL, Dalbeth N, Fransen J, Schumacher HR, Berendsen D, et al. 2015 Gout classification criteria: an American College of Rheumatology/European League Against Rheumatism collaborative initiative. Ann Rheum Dis. 2015;74(10):1789-98.

[2] Estevez-Garcia IO, Gallegos-Nava S, Vera-Pérez E, Silveira LH, Ventura-Ríos $L$, Vancini $G$, et al. Levels of cytokines and MicroRNAs in individuals with asymptomatic hyperuricemia and ultrasonographic findings of gout: A Bench-to-Bedside Approach. Arthritis Care Res. 2018;70(12):1814-21.

[3] Elsaman AM, Muhammad EM, Pessler F. Sonographic findings in gouty arthritis: diagnostic value and association with disease duration. Ultrasound Med Biol. 2016;42(6):1330-6.

[4] Joosten LAB, Crişan TO, Bjornstad P, Johnson RJ. Asymptomatic hyperuricaemia: a silent activator of the innate immune system. Nat Rev Rheumatol. 2020;16(2):75-86.

Acknowledgments: This work was supported by National Natural Science Foundation of China (No. 81571684 to Jiaan Zhu), Peking University People's Hospital Research and Development Funds (RDC2014-02 to Wenting Fan).
Disclosure of Interests: None declared

DOI: 10.1136/annrheumdis-2020-eular.4253

\section{SAT0561 METATARSOPHALANGEAL JOINT MEDIAL COLLATERAL LIGAMENT MEASUREMENT, A NOVEL ULTRASOUND FEATURE OF MONOSODIUM URATE DEPOSITION IN THE JOINT.}

\section{R. Flood ${ }^{1}$, D. Kane ${ }^{2}$, R. Mullan ${ }^{2} .{ }^{1}$ Tallaght University Hospital, Rheumatology,} Dublin, Ireland; ${ }^{2}$ Tallaght University Hospital, Dublin, Ireland

Background: Acute gouty arthritis most commonly initially affects the first metatarsophalangeal joint (MT1). (1) Musculoskeletal ultrasound (US) is a reliable tool for detecting monosodium urate crystal (MSU) deposition in gout and hyperuricemia with validated, ultrasound features of double contour (DC) sign, tophus, and erosions. $(2,3)$ The collateral ligaments of MT1, which originate on the medial and lateral epicondyles of the metatarsals and extend to the proximal phalanx, function to stabilize the joint. (4) While tophus deposition typically occurs between the medial collateral ligament (MCL) and head of MT1, small MSU aggregates may be indistinguishable from surrounding tissue. In this study using US, we propose that an increased vertical depth between the superficial surface of the MCL to cortical surface of MT1 (dMC-MT) is indicative of MSU deposition (see figure 1). The aim was to evaluate associations of dMC-MT with serum uric-acid level (sUA) in a cohort of individuals with hyperuricaemia and non-episodic foot pain. We propose a novel sonographic feature of MSU crystal deposition in the MT joint.

Objectives:

1.) To evaluate the association between sUA and $\mathrm{dMC}-\mathrm{MT}$

2.)To record the presence/absence of classical features of MSU deposition including; double contour sign, erosions and tophi in a cohort of patients with hyperuricaemia and foot pain.

3.) To evaluate the associations between SUA and $\mathrm{dMC}-\mathrm{MT}$ in those withlwithout classical features of MSU deposition (DC, erosion, tophi).

Methods: Following informed consent, hyperuricaemic patients $(n=52)$ underwent bilateral US of the 1MT using LogiqE9 at $15 \mathrm{MHz}$. Features of MSU deposition including DC sign, tophus and juxta-articular erosion were recorded. The dMC-MT was measured as the mean of the perpendicular distance between the superficial surface of the midpoint of the MCL to the MT1 head. Statistical analysis was performed using SPSS V.25 software. Data presented as MEAN \pm S.E unless otherwise indicated.

Results: DC sign, tophus and erosion occurred in $31 \%, 20.7 \%$ and $19 \%$ of cases, respectively. Mean SUA was higher in tophus positive $(540 \pm 36)$ versus non tophus $(470 \pm 16)(p<0.01)$ and erosion positive $(522 \pm 32)$ versus non erosion

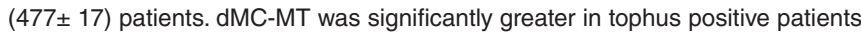
$(0.34 \mathrm{~cm} \pm 0.17 \mathrm{~cm})$ versus non tophus $(0.27 \mathrm{~cm} \pm 0.01 \mathrm{~cm})(p<0.01) . d M C-M T$ was significantly greater in erosion positive patients $(0.31 \mathrm{~cm} \pm 0.18 \mathrm{~cm})$ versus non erosion $(0.28 \mathrm{~cm}+0.01 \mathrm{~cm})(p<0.05)$. In DC negative patients dMC-MT was significantly correlated with increasing sUA $(r=0.34 p=<0.05)$. No correction between dMC-MT and SUA was seen in DC positive patients.

Conclusion: dMC-MT is significantly greater both in patients with tophus and erosions indicating its role as an additional marker of MSU crystal deposition. Furthermore a significant association between dMC-MT and SUA in DC negative patients suggests that dMC-MT may be a more sensitive indicator of early urate deposition in a subset of patients where the earliest site of urate deposition has not occurred directly on to articular hyaline cartilage. dMC-MT may therefore be a sensitive tool for very early urate deposition. Further studies clarifying a role for $\mathrm{AMC}-\mathrm{MT}$ are now required.

References:

[1] Wallace SL, Robinson H, Masi AT, Decker JL, Mccarty DJ, Yü T -f. Preliminary criteria for the classification of the acute arthritis of primary gout. Arthritis Rheum. 1977;20(3):895-900.

[2] Howard RG, Pillinger MH, Gyftopoulos S, Thiele RG, Swearingen CJ, Samuels J. Reproducibility of musculoskeletal ultrasound for determining monosodium urate deposition: Concordance between readers. Arthritis Care Res. 2011;63(10):1456-62.

[3] Stewart S, Dalbeth N, Vandal AC, Rome K. Characteristics of the first metatarsophalangeal joint in gout and asymptomatic hyperuricaemia: $A$ cross-sectional observational study. J Foot Ankle Res. 2015;8(1):1-8.

[4] Finney FT, Cata E, Holmes JR, Talusan PG. Anatomy and Physiology of the Lesser Metatarsophalangeal Joints. Foot Ankle Clin. 2018;23(1):1-7. 